\title{
Non-local regularization of chiral quark models in the soliton sector ${ }^{1}$
}

\author{
Georges Ripka* and Bojan Golli ${ }^{\dagger}$ \\ * Service de Physique Théorique, Centre d'Etudes de Saclay \\ F-91191 Gif-sur-Yvette Cedex, France ${ }^{2}$ \\ ${ }^{\dagger}$ Faculty of Education, University of Ljubljana and J.Stefan Institute, \\ Jamova 39, P.O.Box 3000, 1001 Ljubljana, Slovenia ${ }^{3}$
}

\begin{abstract}
A chiral quark model is described which is regularized in terms of Lorentz invariant non-local interactions. The model is regularized to all loop orders and it ensures the proper quantization of the baryon number. It sustains bound hedgehog solitons which, after suitable centre of mass corrections, can adequately describe the nucleon.
\end{abstract}

\section{SOME SPECIFICITIES OF CHIRAL QUARK MODELS}

This work was done in collaboration with Wojciech Broniowski from Krakow. We consider chiral quark models which encompass three sectors. The vacuum and soliton sectors, which are treated in the mean-field (leading order in $N_{c}$ ) approximation, and the meson sector, which describes the (next to leading order in $N_{c}$ ) vibrations of the vacuum sector. Not all models are applicable to the three sectors. For example, constituent quark models, in which quarks interact with confining forces, cannot describe the vacuum sector, that is, the Dirac sea. However, they can and do describe the excited states of baryons, a thing which the chiral quark models cannot do (except, possibly, the $\Delta$ ) for lack of confinement.

Chiral quark models (nor any of the other low energy quark models) have not been derived from QCD. The only serious attempt to derive them from QCD is the instanton gas model $[1,2]$. In this approach, the chiral quark model is derived by calculating the propagation of quarks in a gas of instantons. A regularized effective theory results, as it should. It predicts both the value of the cut-off and the form of the regulator. The non-local regularization discussed here has the same form as

1) talk presented by G. Ripka at the International Workshop on Hadron Physics "Effective Theories of Low Energy QCD”, Coimbra, Portugal, September 10-15, 1999

2) E-mail: ripka@spht.saclay.cea.fr

3) E-mail: Bojan.Golli@ijs.si 
the one derived from the instanton gas model. Unfortunately, the quark models derived from the instanton structure of the vacuum do not lead to quark or color confinement. This serious limitation serves as a reminder that we have not really succeeded in deriving low energy effective theories from QCD.

Other so-called "derivations" of quark models from QCD involve more guesswork than derivation. Most telling is their inability to derive a regularized model. If infinities appear in an effective theory, one should seek the physical processes which prevent the infinities from occurring. Invoking the roughly $200 \mathrm{MeV}$ QCD cut-off is not a serious argument. Nor does QCD imply in any sense that the quark-quark interaction at low energy should be a one-gluon exchange with a modified gluon propagator. The regularizations used so far in the Nambu Jona-Lasinio type models for example (proper-time regularization being the most commonly used one so far), are nothing but renormalization techniques in which a finite cut-off is maintained. Not only is this arbitrary but such regularizations are flawed with problems.

One might argue that the value of the cut-off should not matter. Indeed it would not if the effective theory consisted, for example, in eliminating some high energy degrees of freedom and using the remaining degrees of freedom to work out the dynamics of low energy phenomena. In such a case, one might expect the cut-off to be much larger than the inverse size of the composite particles and the results not to be sensitive to the cut-off. In chiral quark models, however, this is not the case. The cut-offs required to fit $f_{\pi}$ are about $700 \mathrm{MeV}$, hardly larger than the $\rho$ or the nucleon mass. This is a fact of life, whether we like it or not. One can of course simply discard such models, but better models do not seem to be forthcoming.

\section{THE SOLITON IN THE NON-LOCAL CHIRAL QUARK MODEL}

The non-local chiral quark model is defined by the euclidean action:

$$
I\left(q, q^{\dagger}\right)=\left\langle q\left|\partial_{\tau}+\frac{\vec{\alpha} \cdot \vec{\nabla}}{i}+m\right| q\right\rangle-\frac{G^{2}}{2} \int d_{4} x\left(\langle q|r| x\rangle \beta \Gamma_{a}\langle x|r| q\rangle\right)^{2} .
$$

In this expression, $\Gamma_{a}=\left(1, i \gamma_{5} \tau_{a}\right), q(x) \equiv\langle x \mid q\rangle$ is the quark field, and $r$ is a regulator. The regulator is assumed to be diagonal in momentum space and it has a range which defines an effective euclidean cut-off $\Lambda$. For example, we could take $\left\langle k|r| k^{\prime}\right\rangle=\delta_{k, k^{\prime}} r\left(k^{2}\right)$ with $r\left(k^{2}\right)=e^{-\frac{k^{2}}{2 \Lambda^{2}}}$, where $k$ is a euclidean 4-vector $k_{\mu}=(\omega, \vec{k})$ with $k^{2}=\omega^{2}+\vec{k}^{2}$. The interaction term of the action (1) can be viewed as a contact 4 -fermion interaction involving the delocalized quark fields:

$$
\psi(x)=\langle x|r| q\rangle=\int d_{4} y\langle x|r| y\rangle q(y) .
$$

An action of the form (1) is derived from the instanton gas model of the QCD vacuum $[1,2]$, which predicts a cut-off function of the form: 


$$
r\left(k^{2}\right)=f(k \rho / 2), \quad f(z)=-z \frac{d}{d z}\left(I_{0}(z) K_{0}(z)-I_{1}(z) K_{1}(z)\right)
$$

where $\rho$ is the instanton size. The the cut-off is determined by the inverse instanton size $\rho$. The form (3) has $r(z=0)=1$ and $r(z) \underset{z \rightarrow \infty}{\rightarrow} \frac{9}{2 k^{6} \rho^{6}}$. However, at large euclidean momenta $k$, the form (3) is no longer valid and the cut-off function is dominated by one gluon exchange. It decreases as $\frac{1}{k^{2}}$ (with possible logarithmic corrections) and not as $\frac{1}{k^{6}}$. We find that the fall-off of the regulator at large euclidean $k^{2}$ does not affect the soliton properties very much. For this reason, we have felt free to use various simple forms of cut-off functions, such as a gaussian, which have an additional advantage in that they can be analytically (although arbitrarily) continued to negative values of $k^{2}$. We shall see below that the analytic continuation is required to include the valence orbit. Similar regularization has been used by the Manchester group [3] in the meson and vacuum sectors. Various regularization schemes are reviewed in chapter 6 of Ref. [4].

The euclidean action allows us to calculate the partition function $Z=$ $\int D(a) D\left(a^{\dagger}\right) e^{-I\left(a, a^{\dagger}\right)}$ and the ground state energy $E=-\frac{\partial}{\partial \beta} \ln Z$. The partition function cannot be written in the form $Z=\operatorname{Tr} e^{-\beta H}$ because the regulator in the action (1) prevents us from defining a hamiltonian $H$. We are also unable to quantize the quark fields but we shall see that the baryon number is nonetheless properly quantized.

We work with the equivalent bosonized form of the action:

$$
I(\varphi)=-\operatorname{Tr} \ln \left(\partial_{\tau}+\frac{\vec{\alpha} \cdot \vec{\nabla}}{i}+\beta m+\beta r \varphi_{a} \Gamma_{a} r\right)+\frac{1}{2 G^{2}} \int d_{4} x \varphi_{a}^{2}(x)
$$

in which case the partition function is given by the path integral $Z=\int D(\varphi) e^{-I(\varphi)}$. We refer to $\varphi_{a} \Gamma_{a}=S+i \gamma_{5} \tau_{a} P_{a}$, as the "chiral field" and we say that the chiral field is "on the chiral circle" if, for all $x$, we have $S^{2}(x)+P_{a}^{2}(x)=M_{0}^{2}$, where $M_{0}$ is an $x$-independent constant mass.

We have calculated a localized and time independent stationary point of the action (4), consisting of a chiral field with a hedgehog shape $S(r)+i \gamma_{5} \widehat{x}_{a} \widehat{\tau}_{a} P(r)$ [5]. The shape of the fields and the soliton energy can be calculated in terms of the energies $e_{\lambda}(\omega)$ of the quark orbit. The "Dirac hamiltonian" is diagonal in the energy representation, although it remains energy dependent. The quark orbits $\left|\omega, \lambda_{\omega}\right\rangle$ satisfy the equations :

$$
\partial_{\tau}\left|\omega, \lambda_{\omega}\right\rangle=i \omega\left|\omega, \lambda_{\omega}\right\rangle, \quad\left(\frac{\vec{\alpha} \cdot \vec{\nabla}}{i}+\beta m+\beta r \varphi_{a} \Gamma_{a} r\right)\left|\omega, \lambda_{\omega}\right\rangle=e_{\lambda}(\omega)\left|\omega, \lambda_{\omega}\right\rangle .
$$

The energy of the soliton is: 


$$
E_{\text {sol }}=N_{c} e_{v a l}+\frac{1}{2 \pi} \int_{-\infty}^{\infty} \omega d \omega \sum_{\lambda_{\omega}} \frac{i+\frac{d e_{\lambda}(\omega)}{d \omega}}{i \omega+e_{\lambda}(\omega)}+\frac{1}{2 G^{2}} \int d_{3} x \varphi_{a}^{2}(\vec{x})-v a c .
$$

where $-v a c$. means that we subtract the vacuum energy. In the vacuum, $P=0$, $S=M_{0}$ and there is no valence orbit contribution $e_{v a l}$. The latter is discussed in the next section.

\section{THE QUANTIZATION OF THE BARYON NUMBER AND THE VALENCE ORBIT}

We calculate the baryon number from the Noether current associated to the gauge transformation $q(x) \rightarrow e^{-i \alpha(x)} q(x)$. It turns out to be:

$$
B=-\frac{1}{2 \pi i N_{c}} \int_{-\infty}^{\infty} d \omega \sum_{\lambda_{\omega}} \frac{i+\frac{d e_{\lambda}(\omega)}{d \omega}}{i \omega+e_{\lambda}(\omega)}
$$

The extra term $\frac{d e_{\lambda}(\omega)}{d \omega}$ in the numerator arises from the fact that the regulator $r$ does not commute with $\alpha(x)$. Its effect is to make the residues of all the poles of the quark propagator $\frac{1}{i \omega+e_{\lambda}(\omega)}$ equal to unity. This effectively quantizes the baryon number in a manner which does not seem to be related to the topology of the hedgehog field. ${ }^{4}$ This is most fortunate because, a priori, there is no reason to expect a theory, in which we cannot quantize the quark field, to yield a properly quantized baryon number.

The expression (7) suggests a way to include the valence orbit so as to ensure that the baryon number of the soliton, relative to the vacuum, is equal to unity. We calculate "on-shell" pole of the quark propagator in the hedgehog background field by searching for a solution of the equation $i \omega+\left.e_{\lambda}(\omega)\right|_{\omega=i e_{v a l}}=0$. Because of the regulator, the solutions are scattered all over the complex $\omega$ plane. However, it is well known that, in the local theory, where we set $r=1$, and for a hedgehog field with winding number unity, a well separated bound orbit with grand spin and parity $0^{+}$occurs with energy $e_{v a l}$ close to zero [6]. In the non-local theory, we find that a solution of the equation $\omega=i e_{v a l}(\omega)$ can always be found on the imaginary $\omega$ axis, close to the origin $\omega=0$, and that no other pole occurs in the vicinity. We therefore ensure that the soliton has a baryon number $B=1$ by deforming the integration path over $\omega$ in such a way as to include the contribution of this pole. This requires an analytic continuation of the regulator. Such a continuation is arbitrary but the analytic continuation does not extend as far from the origin as $e_{v a l}$. Indeed, since the soliton size is small, $\vec{k}^{2}>0$ is large and this, on the average, makes $k^{2}=-e_{v a l}^{2}+\vec{k}^{2}$ less negative. Unfortunately however, the form (3) of the regulator, predicted in the instanton model, does not allow any analytic continuation whatsoever, thereby, strictly, prohibiting its use in the soliton calculation.

4) Nor is the soliton stabilized by the topology of the chiral field. 


\section{RESULTS OF SELF-CONSISTENT SOLITON CALCULATIONS}

The model parameters are the coupling constant $G$ appearing in the lagrangian, the cut-off $\Lambda$ appearing in the regulator and the current quark mass $m$. The values of the three parameters are constrained by fitting the pion decay constant $f_{\pi}=93 \mathrm{MeV}$ and the pion mass to $m_{\pi}=139 \mathrm{MeV}$. The expression used to calculate the pion decay constant $f_{\pi}$ is:

$$
f_{\pi}^{2}=2 N_{f} N_{c} M_{0}^{2} \int \frac{d_{4} k}{(2 \pi)^{4}} \frac{r_{k}^{4}-k^{2} r_{k}^{2} \frac{d r_{k}^{2}}{d k^{2}}+k^{4}\left(\frac{d r_{k}^{2}}{d k^{2}}\right)^{2}}{\left(k^{2}+r_{k}^{2} M_{0}^{2}\right)^{2}}
$$

valid in the chiral limit $m \rightarrow 0$ and it is not identical to the Pagels-Stokar formula [7]. This leaves one undetermined parameter which we choose to be the constituent quark mass $M_{0}$ at zero 4-momentum. The pion decay constant $f_{\pi}$ sets the scale. Grossly, soliton energies increase and soliton radii diminish as $f_{\pi}$ increases (see table 2).

Figure 1 shows the soliton energy $E_{\text {sol }}$ as a function of the free parameter $M_{0}$. A soliton is a bound state of $N_{c}=3$ quarks which polarize the Dirac sea. With a gaussian regulator, it is formed if $M_{0} \gtrsim 276 \mathrm{MeV}$, that is, for a sufficiently strong coupling constant $G \gtrsim 4.7 \times 10^{-3} \mathrm{MeV}^{-1}$. The bound state occurs when the energy of the system is lower than the energy $N_{c} M_{q}$ of $N_{c}$ free constituent quarks in the vacuum: $E_{s o l}<N_{c} M_{q}$. The mass $M_{q}$ is the on-shell constituent quark mass, obtained by searching for the pole of the quark propagator in the vacuum. It is the solution of the equation $k^{2}+\left.\left(r_{k}^{2} M_{0}+m\right)^{2}\right|_{k^{2}=-M_{q}^{2}}=0$, which requires an analytic continuation of the regulator to negative values of $k^{2}$. Figure 1 also shows $N_{c} M_{q}$. At the critical value $M_{0} \approx 276 \mathrm{MeV}$, the two curves merge. The contribution $N_{c} e_{v a l}$ of the valence orbit is also shown. At the critical value of $M_{0}$, the energy $e_{v a l}$ of the valence orbit, which is the on-shell mass of a quark propagating in the hedgehog field, becomes a well distinguished bound orbit.

At $M_{0} \approx 309 \mathrm{MeV}$, the curve displaying $N_{c} M_{q}$ on figure 1 abruptly stops. Indeed, for larger values of $M_{0}$, the poles of the quark propagator no longer occur for real values of $k^{2}$. This means that quarks can no longer materialize on-shell in the vacuum. This feature is discussed in chapter 6 of Ref. [4] and it has been considered by several authors as a sign of quark confinement [8-10]. In fact, when a pole of the quark propagator disappears from the real $k^{2}$ axis, it simply moves into the complex plane. Such poles indicate instability of the assumed vacuum state against the addition of a single quark.

However, our calculation shows that, in the background soliton field, the on-shell valence orbit continues to exist and so does the soliton. Unfortunately, the regulator also introduces extra unwanted poles in the propagators of colorless mesons, so that the model does not express color confinement. Similar unwanted poles occur 


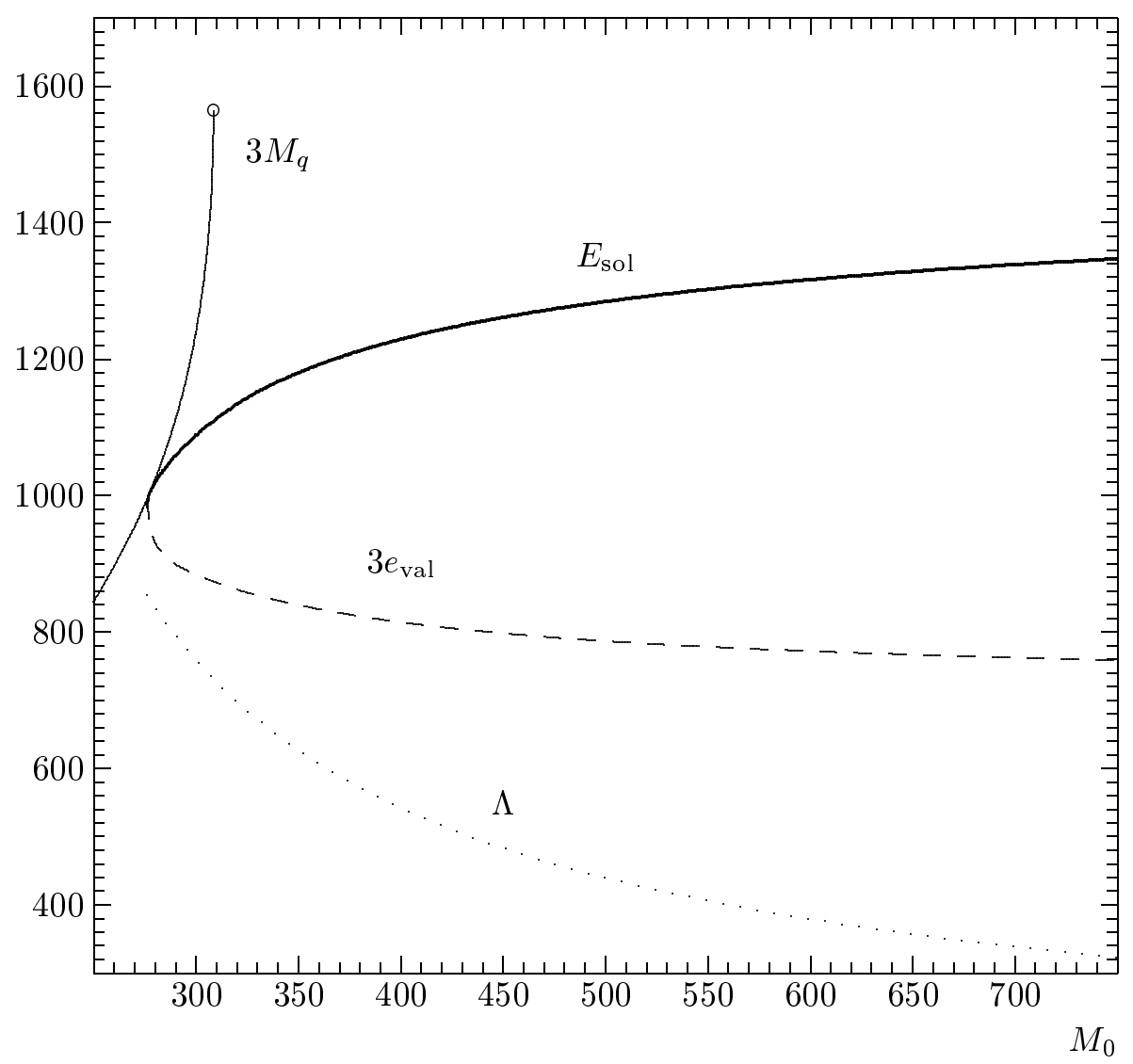

FIGURE 1. The energy of the soliton [in $\mathrm{MeV}$ ] (bold solid line), $N_{c}$ times the free-space quark mass (solid line) and the valence contribution to the soliton energy (dashed line) plotted as functions of the parameter $M_{0}$ [in $\mathrm{MeV}$ ]. A Gaussian regulator is used; $\Lambda$ (dots) is fitted to $f_{\pi}=93 \mathrm{MeV}$.

in proper-time regularization [11]. Our ignorance as how to continue propagators in the complex $k^{2}$ plane reflects our ignorance of the confining mechanism [12].

Apart from the solitons consisting of three valence quarks we find stable solitons consisting of a single valence quark in the background soliton field (see figure 2) as well as of two valence quarks. Similar solutions have been found in the linear sigma model with valence quarks [13].

Figure 3 shows the scalar and pseudoscalar fields $S(x) / M_{0}$ and $P(x) / M_{0}$ of the soliton obtained with several values of $M_{0}$, together with the soliton quark density $\rho(x)$. Note that, within the soliton, the fields do not lie on the chiral circle and $S^{2}(x)+P^{2}(x)<M_{0}^{2}$. Indeed, the pion component $P(x)$ never reaches the values $-M_{0}$. This is a new dynamical result. This is the only calculation, as far we know, in which one can check dynamically whether the chiral field remains or not on the chiral circle. It could not be checked in the renormalized linear sigma model, because close lying Landau poles occur which make the soliton unstable 


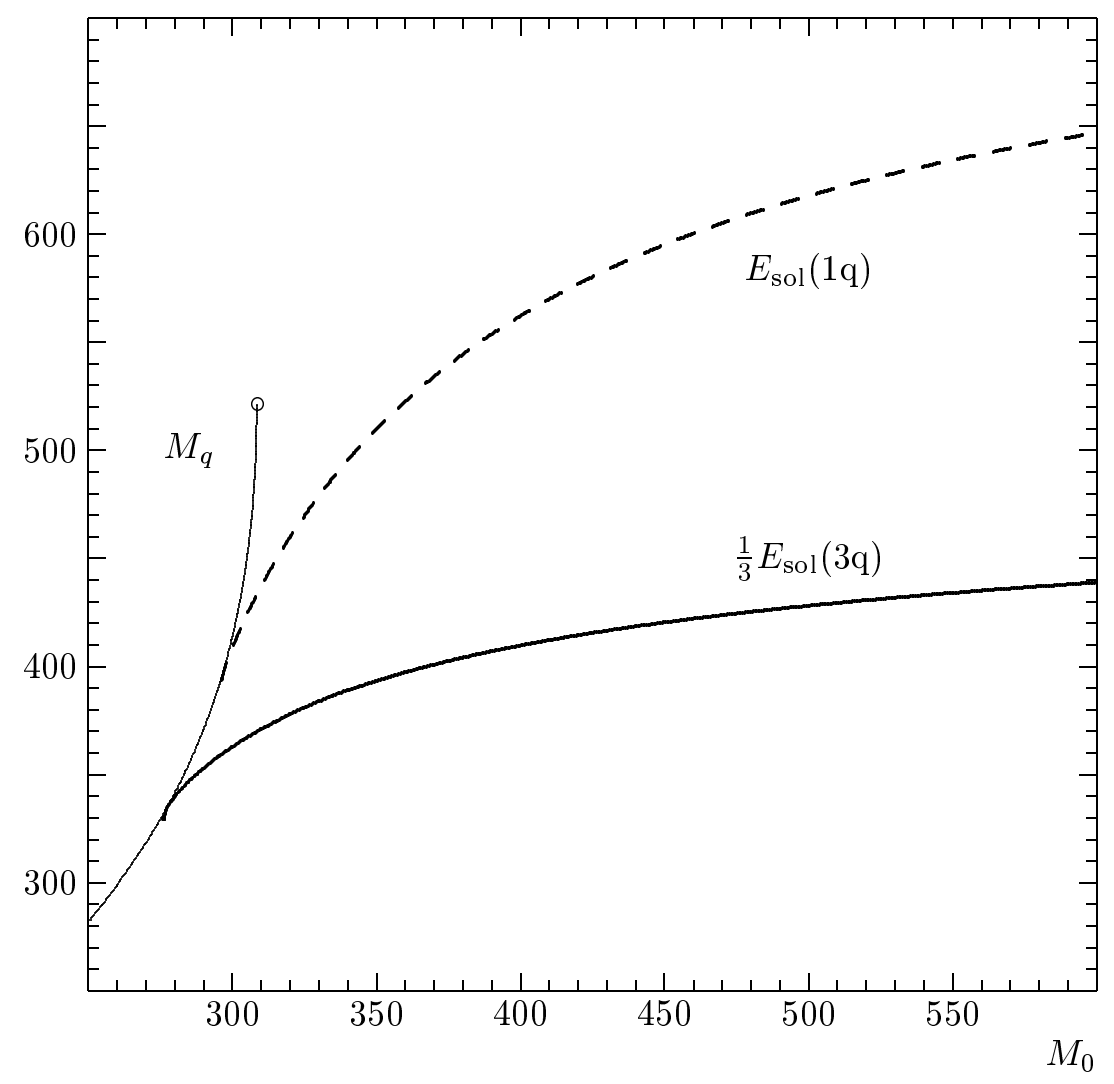

FIGURE 2. The energy per quark $[\mathrm{in} \mathrm{MeV}]$ for the soliton with three valence quarks (bold line), the soliton with one valence quark (dashed line) and the free-space quark mass $M_{q}$ plotted as functions of the parameter $M_{0}[$ in $\mathrm{MeV}]$.

against high gradients in the fields $[14,15]$. It could also not be checked in local theories which use proper-time regularization because, in such theories, the soliton is unstable unless the fields are constrained to remain on the chiral circle $[16,17]$. No such instability occurs with the non-local regularization.

The soliton we obtain with non-local regularization has a structure which lies midway between a Friedberg-Lee soliton [18,19] (in which the pion field has a vanishing classical value), and a Skyrmion [20,21] (in which the chiral field is constrained to remain on the chiral circle). This raises the problem of the collective rotational motion of the soliton. If the deformation in spin and isospin space is stable enough to sustain a rotation without significant distortion, then the $\Delta$ can be described as a rotation of the soliton and the $N-\Delta$ mass splitting can be estimated by cranking. If, however, the deformation is small, the $\Delta$ may be better described as a bound state of quarks with aligned spins and isospins. We have not tackled this problem yet.

Table 1 shows some properties of calculated solitons for various values of the mass parameter $M_{0}$. Rather good values of $g_{A}$ are obtained. The soliton mass and 


\begin{tabular}{|c|c|c|c||c||c|c|c|c|c|}
\hline $\begin{array}{c}M_{0} \\
\mathrm{MeV}\end{array}$ & $\begin{array}{c}\Lambda \\
\mathrm{MeV}\end{array}$ & $\begin{array}{c}m \\
\mathrm{MeV}\end{array}$ & $\begin{array}{c}\langle\bar{q} q\rangle^{1 / 3} \\
\mathrm{MeV}\end{array}$ & $\begin{array}{c}1 / G \\
\mathrm{MeV}\end{array}$ & $\begin{array}{c}e_{\text {val }} \\
\mathrm{MeV}\end{array}$ & $\begin{array}{c}E_{\text {Dirac }} \\
\mathrm{MeV}\end{array}$ & $\begin{array}{c}E_{\text {sol }} \\
\mathrm{MeV}\end{array}$ & $\begin{array}{c}\left\langle r^{2}\right\rangle^{1 / 2} \\
\mathrm{fm}\end{array}$ & $g_{A}$ \\
\hline 300 & 760 & 7.62 & -215 & 182 & 295 & 2360 & 1088 & 1.32 & 1.28 \\
350 & 627 & 10.4 & -200 & 140 & 280 & 1715 & 1180 & 1.04 & 1.16 \\
400 & 543 & 13.2 & -185 & 113 & 272 & 1433 & 1229 & 0.97 & 1.14 \\
450 & 484 & 15.9 & -173 & 94 & 266 & 1275 & 1261 & 0.96 & 1.12 \\
\hline
\end{tabular}

TABLE 1. Properties of self-consistent soliton solutions obtained with a gaussian regulator.

energies need to be corrected for spurious centre of mass motion (see table 2).

The fields which describe the soliton break translational symmetry. The center of mass of the system is not at rest and it makes a spurious contribution both to the energy and to the mean square radius (more generally, to the form factor). This spurious contribution is not measured and it should be subtracted from the calculated values. The subtraction occurs at the next to leading order (in $N_{c}$ ) approximation. A rough estimate can be obtained from an oscillator model. If $N_{c}$ particles of mass $m$ move in a $1 s$ state of a harmonic oscillator of frequency $\hbar \omega$, the centre of mass of the system is also in a $1 s$ state and it contributes $\frac{3}{4} \hbar \omega=$ $\left\langle P^{2}\right\rangle / 2 N_{c} m$ to the energy. We have therefore corrected the soliton energies by subtracting $\left\langle P^{2}\right\rangle / 2 E_{\text {sol }}$ from the calculated energy. Furthermore, in the oscillator model, the center of mass contributes a fraction $\frac{1}{N_{c}}$ of the mean square radius, so that we have corrected the mean square radius by multiplying the calculated value by a factor equal to $\left(1-\frac{1}{N_{c}}\right)$.

Table 2 shows the result. The soliton energies and radii are then considerably closer to the experimental values observed in the nucleon.

\begin{tabular}{|c|c|c|c|c|}
\hline$M_{0}$ & $\overline{E_{\text {sol }}}$ & $\left\langle r^{2}\right\rangle_{s o l}$ & $E_{\text {corr }}$ & $\left\langle r^{2}\right\rangle$ \\
\hline $\mathrm{MeV}$ & $\mathrm{MeV}$ & $\mathrm{fm}^{2}$ & $\mathrm{MeV}$ & $\mathrm{fm}^{2}$ \\
\hline 300 & 1088 & 1.7 & 965 & 1.1 \\
\hline 350 & 1180 & 1.08 & 990 & 0.72 \\
\hline 400 & 1229 & 0.94 & 1000 & 0.62 \\
\hline 450 & 1261 & 0.92 & 980 & 0.61 \\
\hline $450^{*}$ & 1458 & 0.69 & 1200 & 0.43 \\
\hline
\end{tabular}

TABLE 2. Elimination of spurious c.m. motion. Gaussian regulator, $\Lambda$ fitted to $f_{\pi}=93 \mathrm{MeV} ;{ }^{*} \Lambda$ fitted to $f_{\pi}=1.25 \times 93 \mathrm{MeV}$.

\section{CONCLUSION: WHY TAKE THE TROUBLE?}

The non-local regularization effectively cuts out of the quark propagators the 4momenta which are larger than the cut-off. The non-local regularization makes the 


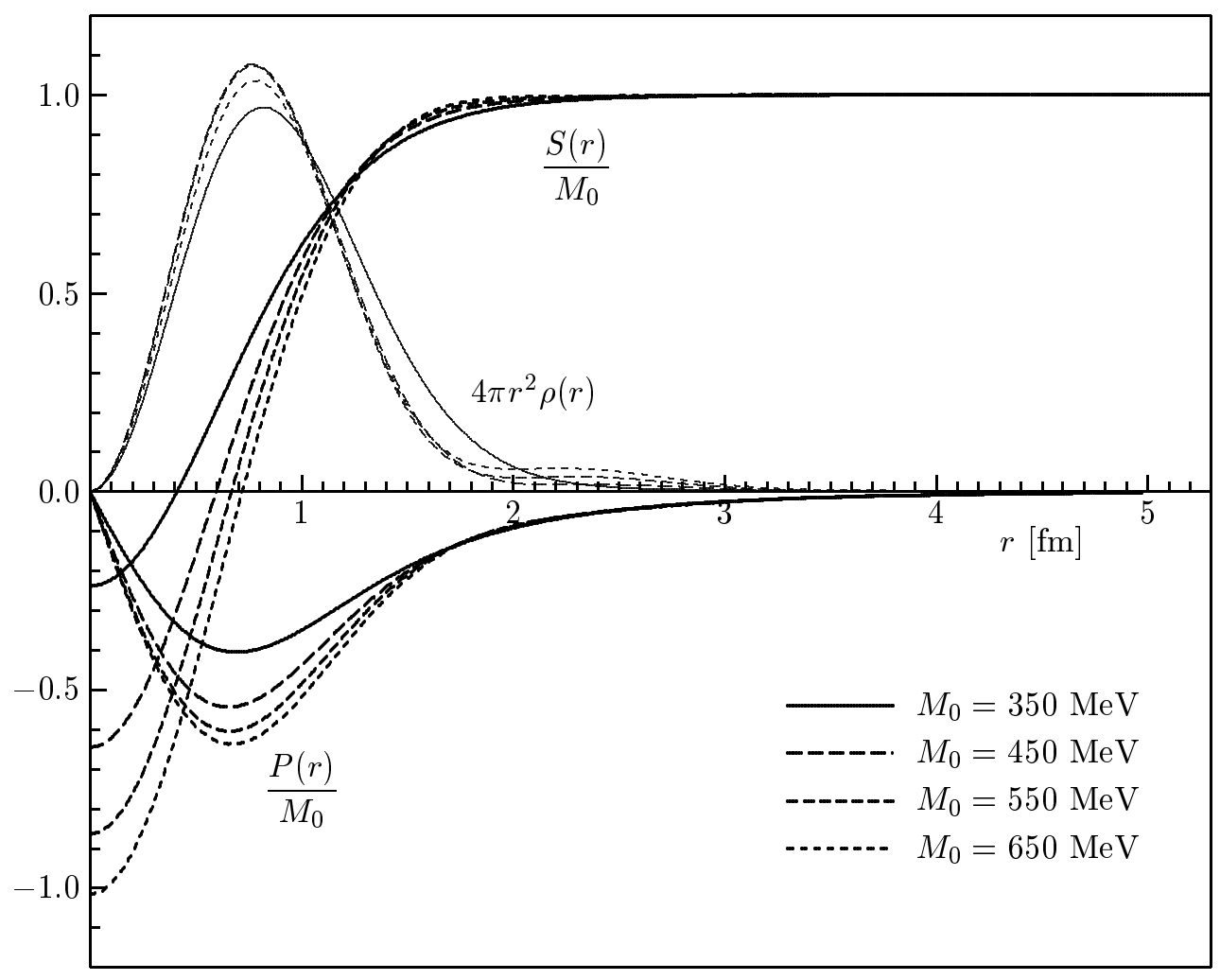

FIGURE 3. Self consistently determined fields and baryon densities $\left(4 \pi r^{2} \rho\right)$ for various values of $M_{0}$; a gaussian regulator is used.

theory finite at all loop orders. The simpler proper-time and Pauli-Villars regularization schemes regularize the quark loop only and they require extra independent cut-offs when next to leading order meson loops are included. Both the real and the imaginary parts of the action are regularized, while the anomalous properties remain independent of the cut-off [22-24], and the baryon number remains properly quantized. In proper time and Pauli-Villars regularization schemes only the real part of the action is regularized and the imaginary part is left unregularized in order to enforce correct anomalous processes. Why not limit the 3-momenta of the quarks, thereby avoiding unwanted extra poles in the propagators? Breaking Lorentz covariance in the meson sector is annoying in that it requires to boost composite particles calculated in their rest frame.

\section{REFERENCES}

1. Diakonov, D. I. and Petrov, V. Y., Nucl. Phys. B272, 457 (1986).

2. Shuryak, E., Nucl. Phys. B203, 93,116,140 (1982).

3. Plant, R. S. and Birse, M. C., Nucl. Phys. A628, 607 (1998). 
4. Ripka, Georges, Quarks Bound by Chiral Fields, Oxford University Press, Oxford 1997.

5. Broniowski, W., Golli, B. and Ripka, G., Phys. Lett. B437, 24 (1998).

6. Kahana, S. and Ripka, G., Nucl. Phys. A429, 462 (1984).

7. Pagels, H. and Stokar, S., Phys. Rev. D20, 2947 (1979).

8. Buballa, M. and Krewald, S., Phys. Lett. B294, 19 (1992).

9. Roberts, C. D. and Williams, A. G., Prog.Part. And Nucl. Phys. 33, 475 (1994).

10. Bowler, R. D. and Birse, M. C., Nucl. Phys. A582, 655 (1995).

11. Nikolov, E. N., Broniowski, W., Ripka, G. and Goeke, K., Zeit. Phys. A354, 421 (1996).

12. Reusch, H., Stingl, M., Häbel, U., Könning, R. and Wizard, S., Zeit. Phys. A336, 423,435 (1990).

13. Golli, B. and Rosina, M., Phys. Lett. B393, 161 (1997).

14. Ripka, G. and Kahana, S., Phys. Rev. D36, 1233 (1987).

15. Perry, R., Phys. Lett. B199, 489 (1987).

16. Grümmer, F., Sieber, P., Meissner, Th. and Goeke, K., Nucl. Phys. A547, 459 (1992).

17. Wünsch, R., Sieber, P., Grümmer, F., Meissner, Th., Ripka, G. and Goeke, K., Phys. Lett. B299, 183 (1993).

18. Lee, T. D., Particle Physics and Introduction to Field Theory, Harwood Academic Press (New-York) 1981.

19. Wilets, L., Nontopological solitons, World Scientific, Singapore, 1989.

20. Skyrme, T. H. R., Nucl. Phys. 31, 556 (1962).

21. Holzwarth, G., editor., Baryons as Skyrme Solitons, World Scientific, Singapore, 1993.

22. Cahill, R. T., Roberts, C. D. and Praschifka, J., Ann. Phys. (NY) 188, 20 (1988).

23. Terning, J., Holdom, B., and Verbeek, K., Phys. Lett. B232, 351 (1989).

24. Ball, R. D. and Ripka, G., in Souza, C., Fiolhais, C., Fiolhais, M. and Urbano, J. N. editors, Many Body Physics (Coimbra 1993), World Scientific, 1993. 